\title{
Salud mental de adolescentes españoles según variables contextuales y horas de uso de internet *
}

\section{Mental Health in Spanish Adolescents According Contextual Variables and Hours of internet Use}

\author{
Fernando Fajardo Bullón $^{\text {a }}$ \\ Universidad de Extremadura, España \\ ORCID: http://orcid.org/0000-0003-0198-0170 \\ Beatriz Burguillo Valverde \\ Universidad de Extremadura, España \\ Benito León Del Barco \\ Universidad de Extremadura, España \\ ORCID: http://orcid.org/0000-0003-0061-9498 \\ Elena Felipe Castaño \\ Universidad de Extremadura, España \\ ORCID: http://orcid.org/0000-0001-6167-877X
}

\footnotetext{
a Autor de correspondencia. Correo electrónico: fernandofajardo@unex.es
}

Para citar este artículo: Fajardo Bullón, F., Burguillo Valverde, B., León-del-Barco, B., \& Felipe Castaño, E. (2019. Salud mental de adolescentes españoles según variables contextuales y horas de uso de internet. Universitas Psychologica, 18(2), 1-12. https://doi.org/ 10.11144/Javeriana.upsy18-2.smae

\section{RESUMEN}

El análisis de la salud mental en adolescentes es un tema actual de interés internacional. El objetivo de esta investigación fue analizar la salud mental de los menores españoles, evaluada mediante la puntuación total de dificultades del Cuestionario de Capacidades y Dificultades (SDQ), en función del número de horas de conexión a internet y otras variables contextuales, como tipo de centro, sexo, curso y edad. La metodología utilizada fue un estudio ex post facto, de corte transversal, con una muestra formada por 430 estudiantes de España de 12 a 17 años. Los resultados muestran una asociación significativa entre la salud mental de los menores, el número de horas de uso de internet $\left(\chi^{2}=10.2 ; p=0.017\right)$ y la edad $\left(\chi^{2}=11.86 ; p=0.037\right)$. Sin embargo, esta asociación no se encontró con el tipo de centro educativo $(U=20026, p=0.418)$, el sexo $(\mathrm{U}=18638, p=0.082)$ y el curso académico $\left(\chi^{2}=1.62, p=0.655\right)$. Se puede concluir que aquellos menores conectados a internet más de cinco horas al día presentaron mayores puntuaciones en dificultades en salud mental. Del mismo modo, los menores con edades entre 15 y 17 años, obtuvieron mayores puntaciones en dificultades en salud mental en comparación con los más jóvenes, de 12 años. Finalmente, se sugiere realizar labores de prevención en salud mental focalizadas en menores en la adolescencia tardía y que se conecten a internet más de cinco horas al día.

Palabras clave

salud mental; internet; variables contextuales; adolescente; España.

\section{ABSTRACT}

The analysis of adolescent mental health is a current issue of international research interest. The purpose of this research was to analyze the mental 
health of Spanish children, assessed by the total difficulty score of The Strengths and Difficulties Questionnaire (SDQ), according to the time they remain connected to the internet and other contextual variables, such as the type of center, sex, school year and age. The methodology used was an ex post facto, cross-sectional study, with a sample of 430 students from Spain aged 12 to 17 years. Results show a significant association between the total score of difficulties in mental health with the amount of hours of internet use $\left(\chi^{2}=10.2 ; p=0.017\right)$ and age $\left(\chi^{2}\right.$ $=11.86 ; p=0.037)$. However, this association was not significant regarding the type of center $(\mathrm{U}=20026, p=$ $0.418)$, gender $(U=18638, p=0.082)$ and school year $\left(\chi^{2}=1.62, p=0.655\right)$. In conclusion, those adolescents who spent more than 5 hours per day using internet had higher scores in mental health difficulties. In the same way, adolescents aged 15 to 17 years had higher scores in mental health difficulties compared to the youngest ones (12 years old). Finally, the authors recommend to focus the prevention and mental health policies in minors who are in a late adolescent and spend more than 5 hours per day connected to internet.

Keywords

mental health; internet; contextual variables; adolescent; Spain.

Los cambios que han provocado el uso de internet y las nuevas tecnologías en los adolescentes ha supuesto un creciente interés en el campo de la investigación sobre la salud mental (Rodríguez \& Fernández, 2014), donde mayormente se ha analizado su impacto en los menores (Cloquell, 2015; El Asam, Samara, \& Terry, 2019). Esta variable es de gran relevancia, tal y como lo demostró Viñas en 2009 en la ciudad de Girona (España), donde un $6.7 \%$ de la muestra dedicaba más de 30 horas a la semana a internet (una media superior a las cuatro horas diarias) y un $10.2 \%$ se mantuvo conectado a partir de la medianoche. Si se tienen en cuenta los resultados del estudio realizado por la Asociación para la Investigación de Medios de Comunicación (AIMC, 2017), cuyo trabajo cuenta con una muestra de 14822 individuos de 14 años o más, se observa como un $42.6 \%$ de esta dedicaba más de cuatro horas a internet al día, seguidos de un $26.4 \%$ entre dos y cuatro horas, un $17.9 \%$ entre una y dos horas y un 13.1 $\%$ menos de una hora (1\%). Con estos datos se puede avalar que la mayor parte de la muestra nacional española usa constantemente internet y las TIC.

Aunque las nuevas tecnologías poseen una serie de beneficios para las tareas de la vida diaria de los jóvenes (Echeburúa, 2012), cada vez hay más estudios que analizan los problemas y riesgos que puede acarrear un uso excesivo de las mismas (Işik \& Ayaz Alkaya, 2017), sobre todo en los adolescentes. Entre estos riesgos podemos encontrar el aumento del sedentarismo y el aislamiento, problemas de socialización, de sueño y alimentación (Golpe, Isoma, Gómez, \& Rial, 2017). Así, Marciales y Cabra (2011) definen el uso problemático de internet como:

[...] el uso indiscriminado de este medio a cualquier hora del día, y todas las semanas, con mayor afectación de las relaciones interpersonales y surgimiento de problemas de interacción en la vida cotidiana, en comparación con quienes hacen un uso moderado. (p. 859)

Una vez analizado el uso problemático de internet, este tiene que ser valorado para conocer cómo afecta al bienestar psicológico del adolescente (Martino, 2014), tema de gran interés en el campo de investigación en salud mental, sobre el que aún queda bastante por estudiar, y que se percibe en la gran cantidad de instrumentos que se puede encontrar para su medición a lo largo de la historia reciente (Fajardo-Bullón et al., 2016). De entre todos ellos, en el presente estudio se ha seleccionado el Cuestionario de Capacidades y Dificultades ([SDQ]; Goodman, 1997), con el que se puede analizar mejor la conducta del niño y sus alteraciones (Fenollar-Cortés, Calvo-Fernández, García-Sevilla, \& Cantó-Díez, 2016; Mata, Suárez, Torres, Carro, \& Ortega, 2009), además de presentar evidencias de validez para su utilidad en el contexto escolar y clínico (Ortuño-Sierra, Fonseca-Pedrero, Inchausti, \& Sastre i Riba, 2016).

Este mismo instrumento ha sido utilizado a nivel nacional por diferentes autores (FajardoBullón, León-del-Barco, Felipe-Castaño, \& Santos, 2012; Fajardo-Bullón, Rasskin-Gutman, Felipe, Santos, \& León-del-Barco, 2017; Fonseca-Pedrero, Paino, Lemos-Giráldez, \& 
Muñiz, 2011; León-del-Barco, Fajardo-Bullón, Mendo-Lázaro, Rasskin-Gutman, \& IglesiasGallego, 2018; Ortuño-Sierra, Fonseca-Pedrero, Paino, \& Aritio-Solana, 2014). Asimismo, también ha sido utilizado en otros estudios a nivel internacional, tales como Finlandia (Koskelainen, Sourander, \& Vauras, 2001), Noruega (Van Roy, Grøholt, Heyerdahl, \& Clench-Aas, 2006), China (Yao et al., 2009) y Holanda (Muris, Meesters, \& Van den Berg, 2003), entre otros. La mayoría de las investigaciones que utilizan en sus evaluaciones el cuestionario SDQ trabajan con las variables sexo y edad. En cuanto al sexo, diferentes estudios mostraron diferencias significativas, donde las mujeres puntuaron más alto en dificultades de salud mental (Koskelainen et al., 2001; Ortuño-Sierra et al., 2014). En los estudios de Arman, Amel y Maracy (2013) y Fonseca-Pedrero et al. (2011), las mujeres también tuvieron mayores puntuaciones en dificultades, aunque no se encontraron diferencias significativas. En cambio, Rothenberger et al. (2008) y Yao et al. (2009) encontraron en sus resultados una mayor puntuación de dificultades en los hombres.

Respecto a la variable edad, gran parte de los estudios muestran una mayor puntuación de dificultad en salud mental en aquellos adolescentes entre los 15 y 18 años frente a la adolescencia temprana con 11-14 años (Arman et al., 2013; Ortuño-Sierra et al., 2014; Yao et al., 2009). Sin embargo, otros autores como FonsecaPedrero et al. (2011) presentan puntuaciones superiores en el intervalo de menor edad de 14-15 años frente al de mayor de 16-17 años.

Por otra parte, acerca de las variables curso académico y tipo de centro apenas se han encontrado estudios que los relacionen con la salud mental. Zubeidat, FernándezParra, Ortega, Vallejo y Sierra (2009) realizaron un estudio sobre el análisis de las características psicosociales y psicopatológicas de una muestra de 961 adolescentes españoles teniendo en cuenta el curso escolar, donde los alumnos de Ciclos Formativos puntuaron más alto en depresión, ansiedad, conducta agresiva y problemas de relación, que aquellos pertenecientes a Educación Secundaria y Bachillerato. En cuanto a la variable tipo de centro, Klanšček, Žiberna, Korošec, Zurc y Albreht (2014) llevaron a cabo un estudio en Eslovenia, donde se señalaba que el tipo de centro ejerce una función significativa en la salud mental de los adolescentes. Dada la escasez de estudios en relación con estas dos variables, en el presente estudio se analizará si la salud mental del adolescente puede variar en función del curso académico o tipo de centro en el que esté matriculado.

Por último, el objetivo de nuestra investigación fue analizar la salud mental de los menores españoles, evaluada mediante la puntuación total de dificultades del cuestionario SDQ, en función del número de horas de conexión a internet, y en relación con otras variables contextuales, tales como el tipo de centro, el sexo, el curso y la edad.

\section{Método}

\section{Participantes}

La muestra de la investigación se compuso por un total de 430 estudiantes de Educación Secundaria Obligatoria de las ciudades de Badajoz y Mérida (España), de tres centros públicos y dos concertados, durante el curso académico 2014-2015. Un $48.9 \%$ eran del sexo masculino y un $51.1 \%$ del sexo femenino. El rango de edad de los participantes oscilaba entre los 12 y los 17 años, con una edad promedio de 14.21 años $(D E=1.34)$. La muestra se obtuvo a partir del número total de alumnos de Educación Secundaria Obligatoria de las ciudades de Mérida y Badajoz, de centros públicos y privados $(\mathrm{N}=$ 8 965). A continuación, se calculó el tamaño muestral para nuestro estudio en función del total de la población, donde se obtuvo como resultado un $n$ por encuestar de 375 alumnos, cuya muestra final se incrementó a 430 alumnos, distribuidos de la siguiente manera: $17.9 \%$ alumnos de centros públicos de Mérida, $23 \%$ alumnos de centros concertados de Mérida, $34.2 \%$ alumnos de centros públicos de Badajoz y $24.9 \%$ alumnos de centros concertados de Badajoz. 


\section{Instrumentos}

Cuestionario de datos sociodemográficos recoge datos como la edad, el sexo, centro de estudio, y preguntas relacionadas con el uso de las nuevas tecnologías.

\section{Cuestionario de Capacidades y Dificultades ([SDQ-Cas]; Goodman, 1997)}

En la presente investigación, se utilizó la versión de autoinforme para menores de 11 a 17 años (http://www.sdqinfo.com). Con este instrumento se pueden valorar distintos problemas comportamentales y emocionales vinculados con la salud mental en los últimos seis meses. Está compuesto de 25 ítems que conforman cinco escalas (con cinco ítems cada una). La suma de las cuatro escalas forman la puntuación total de dificultades (Síntomas emocionales, Problemas de conducta, Hiperactividad y Problemas con compañeros), mientras que la quinta escala alude a Conductas prosociales. Cada uno de los ítems que forman el instumento se evalúa con tres opciones de respuesta: no es verdad, es verdad a medias $y$ verdaderamente sí, puntuándose con 0,1 y 2 , respectivamente. Además, cinco de los ítems se puntúan a la inversa, es decir, 2 (no es verdad), 1 (es verdad a medias) y 0 (verdaderamente sí), cuyos ítems son el 7, 11, 14, 21 y 25 . La puntuación total de dificultades se halla entre 0 y 40 puntos (Fonseca-Pedrero et al., 2011; Rodríguez \& Pérez, 2012).

Asimismo, en la versión autoinforme para niños, se establecen unos puntos de corte para la puntuación total de dificultades, considerando una situación normal a aquellas que oscilan entre 0 y 15 y una situación límite y/o anormal entre 16 y 40 (Ortuño-Sierra et al., 2014). Por último, cabe señalar que en la versión autoinforme para adolescentes españoles, la consistencia interna basada en el $\alpha$ de Cronbach es de 0.84 para la puntuación total de dificultades. En cambio, para cada una de las escalas del cuestionario está entre 0.71 y 0.75 (Ortuño-Sierra, Chocarro, FonsecaPedrero, Sastre i Riba, \& Muñiz, 2015). En la muestra de estudio se obtuvo un $\alpha$ de Cronbach de 0.753 para la puntuación total de dificultades. Se calculó la medida de adecuación muestral $\mathrm{KMO}$ y se obtuvo un valor de 0.741 ( $>0.5$ ). El valor del nivel de significación de la prueba de esfericidad de Bartlett fue $0(<0.05)$. Por último, obtuvo una fiabilidad compuesta $(\mathrm{CF})$ de $0.743(>0.7)$. Por tanto, se obtuvieron una confiabilidad y validez adecuadas.

\section{Procedimiento}

La presente investigación es un estudio ex post facto (de tipo transversal), en el que se emplea la encuesta para la adquisición de evidencia empírica (Montero \& León, 2007). La aplicación de los cuestionarios se realizó en el curso de dos meses. Primero, se contactó con los centros implicados en la investigación para presentar y explicar el estudio y la forma de participación. Cuando estos dieron su aprobación, se procedió a la cumplimentación de los cuestionarios en una sesión de clase o en horario de tutoría, según la disponibilidad horaria que ofrecía cada centro. Se explicó a los alumnos el objetivo del estudio, así como la confidencialidad de los datos, quienes respondieron de forma voluntaria y anónima.

\section{Análisis de datos}

Una vez recogidos los datos, se realizaron los contrastes de normalidad para la variable puntuacion total de dificultades, mediante la prueba de Kolmogorov-Smirnov $(Z=1.96$; $p$ $=0.001)$. Al no cumplirse el principio de normalidad, se procedió a la realización de pruebas no paramétricas. En primer lugar, se efectuó un análisis descriptivo de la muestra y, a continuación, las pruebas para muestras independientes de $U$ de Mann-Whitney y Kruskal-Wallis. Además, se llevaron a cabo dos análisis de varianza (ANOVA) de un factor, para conocer entre qué grupos de horas de conexión y de edad se daban las diferencias en la variable puntuación total de dificultades en salud mental; todo ello a través del programa estadístico SPSS 21.0. 


\section{Resultados}

A continuación, se muestra un análisis descriptivo de las diferentes variables contextuales que se trabajan en este estudio. La muestra estaba formada por estudiantes de 12 a 17 años de edad $(M=14.21 ; D E=$ 1.34). Un $48.9 \%$ eran chicos frente a un 51.1 $\%$ de chicas, distribuidos en diferentes cursos

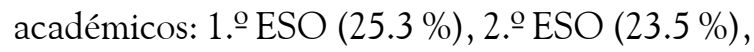
3.․ㅡ ESO (24.9\%) y 4.․ ESO (26.3\%). El 52.1 $\%$ de los adolescentes se encontraba matriculado en centros públicos, mientras que el $47.9 \%$, en centros concertados.

Salud mental y horas de conexión a internet

Para el análisis de la salud mental y las horas de conexión a internet se utilizaron pruebas no paramétricas de Kruskal-Wallis $\left(\chi^{2}\right.$ $=8.57, g l=3, p=0.036)$, obteniéndose diferencias estadísticamente significativas. $\mathrm{Al}$ haber encontrado diferencias en las pruebas no paramétricas, se procedió a realizar la prueba paramétrica de ANOVA de un factor. A través de esta prueba $(F=3.54, g l$ $=3, p=0.015)$, se obtuvieron diferencias estadísticamente significativas entre el tiempo de conexión y la puntuación total de dificultades, por lo que se aplicaron pruebas post hoc mediante el procedimiento de Bonferroni. Para una mejor visualización de dicho análisis, se expone una serie de figuras donde se ofrecen las puntuaciones medias en la variable total de dificultades en salud mental y, por otro lado, se incluyen en una circunferencia aquellos grupos que en el análisis post hoc no obtuvieron diferencias significativas. Se puede observar en la Figura 1, cómo el grupo de tiempo de conexión de más de cinco horas al día presenta diferencias en la puntuación total de dificultades con el grupo de menos de una hora al día.

\section{Figura 1}

Agrupación de las horas de conexión a internet de los menores donde no existen diferencias significativas en las medias de la puntuación total de dificultades del cuestionario SDQ.

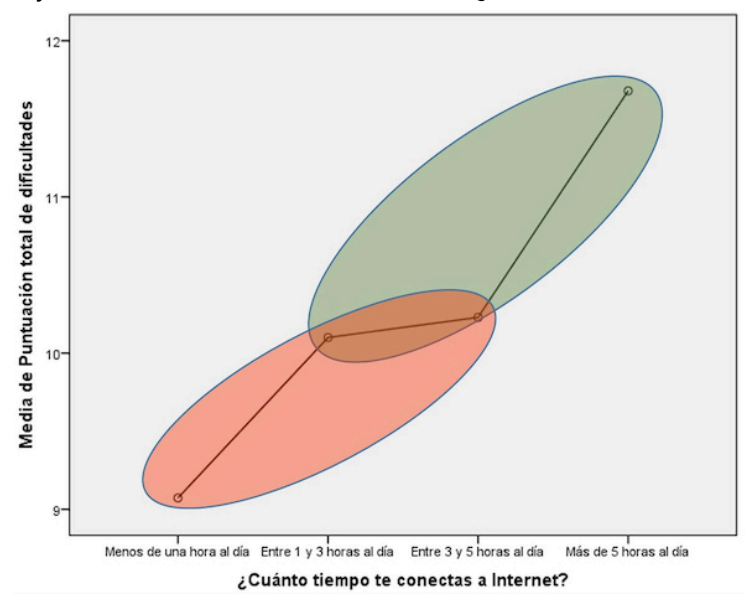

Una vez se observa el aumento conjunto del tiempo de conexión a internet y la puntuación en dificultades en salud mental, no teniendo por qué llegar esta puntuación a niveles de patología, se pasó a analizar la relación que se produce cuando se establecen puntos de corte para el diagnóstico de problemas de salud mental. Para ello, se agruparon los menores según los puntos de corte del total de dificultades del cuestionario SDQ: situación normal, con puntuaciones entre 0 y 15 , y por otro lado, aquellos menores que se encontraban en una situación límite y anormal (entre 16 y 40). Como se puede apreciar en la Tabla 1, se encuentra una asociación significativa entre el número de horas de uso de internet y la salud mental de los menores agrupados según los puntos de corte $\left(\chi^{2}=10.2, g l=3, p=0.017\right)$.

\section{Tabla 1}

Tabla de contingencia entre alumnado con una salud mental normal, límite y/o anormal y tiempo de conexión a internet

\begin{tabular}{|c|c|c|c|c|c|c|}
\hline \multirow{2}{*}{$\begin{array}{c}\text { Conexión a } \\
\text { internet }\end{array}$} & \multicolumn{2}{|c|}{ Salud mental } & \multirow{2}{*}{$\begin{array}{c}\% \\
\text { Total }\end{array}$} & \multirow[b]{2}{*}{$x^{2}$} & \multirow[b]{2}{*}{$g l$} & \multirow[b]{2}{*}{$p$} \\
\hline & $\begin{array}{c}\% \\
\text { Normal }\end{array}$ & $\begin{array}{c}\text { \% Límite } \\
\text { y/o anormal }\end{array}$ & & & & \\
\hline Menos de 1 hora & 12.8 & 0.7 & 13.5 & & & \\
\hline Entre 1 y 3 horas & 31.2 & 5.7 & 36.9 & & & \\
\hline Entre 3 y 5 horas & 20.6 & 2.9 & 23.6 & 10.2 & 3 & 0.017 \\
\hline Más de 5 horas & 19.9 & 6.1 & 26 & & & \\
\hline Total & 84.5 & 15.5 & 100 & & & \\
\hline
\end{tabular}


En la Figura 2, se observa que en aquellos menores con una situación límite y/o anormal en salud mental, el porcentaje más representativo en la variable horas de conexión a internet es el de más de cinco horas al día, mientras que para los que se encontraban en una situación normal, el porcentaje mayor es entre una y tres horas al día. Así, es posible señalar que parece existir una asociación entre pasar más horas en conexión con internet y padecer una situación límite y/o anormal en salud mental.

\section{Figura 2}

Gráfico de barras en función del tiempo de conexión a internet y la salud mental de los menores.

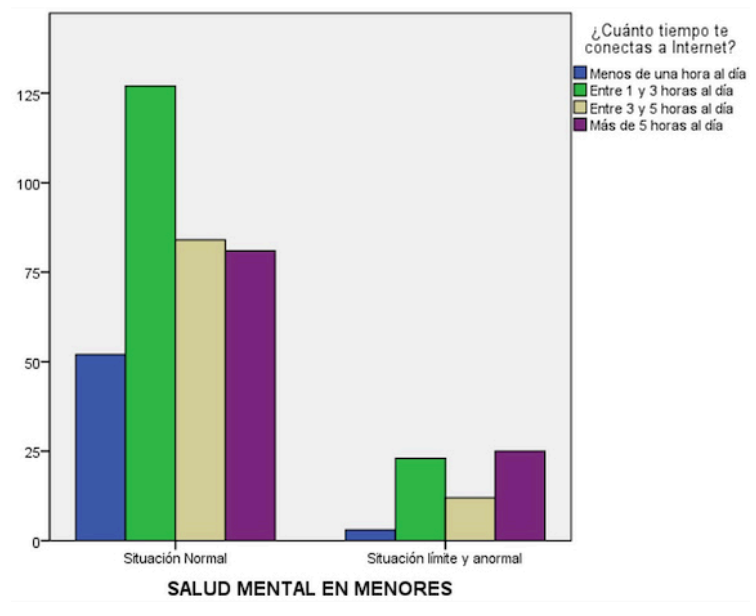

Salud mental y otras variables contextuales (tipo de centro, sexo, curso académico y edad)

A partir de pruebas no paramétricas, se puede señalar que no existen diferencias estadísticamente significativas entre la puntuación total de dificultades y el tipo de centro $(U=20026, p=0.418)$. Lo mismo sucede con el sexo $(U=18638, p=0.082)$ y el curso académico $\left(\chi^{2}=1.62, g l=3, p=0.655\right)$.

Por otro lado, mediante pruebas no paramétricas de Kruskal-Wallis, se obtuvieron diferencias estadísticamente significativas entre la edad y la puntuación total de dificultades $\left(\chi^{2}=11.86, g l=5 ; p=0.037\right)$. Al encontrar diferencias significativas en las pruebas no paramétricas, se procedió a realizar también la prueba paramétrica de ANOVA de un factor. A través de esta prueba $(F=2.35, g l=5$, $p=0.04)$, se obtuvieron también diferencias estadísticamente significativas y se aplicaron pruebas post hoc mediante el procedimiento de DMS. Como se puede observar en la Figura 3, los alumnos de 12 años $(M=8.71, D E=4.81)$ presentaron diferencias en la puntuación total de dificultades con los demás grupos de edad, excepto con los de 14 años $(M=9.83, D E=$ 5.24), por lo que el grupo de alumnos de 12 años presenta diferencias con los de 13 años $(\mathrm{M}=$ $10.58, D E=5.16), 15$ años $(M=11.23, D E=$ 5.24), 16 años $(M=11.18, D E=6.07)$ y 17 años $(\mathrm{M}=11.88, \mathrm{DE}=3.72)$.

\section{Figura 3}

Agrupación de las edades de los menores donde no existen diferencias significativas en las medias de la puntuación total de dificultades del cuestionario SDQ.

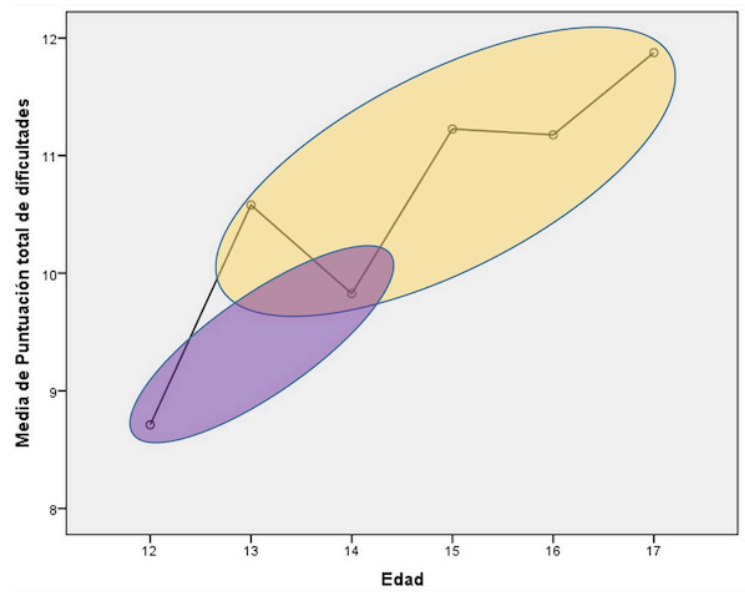

\section{Discusión}

Los resultados obtenidos muestran como más de un $25 \%$ de la muestra de adolescentes se conectaba a internet más de cinco horas al día. Además, en este estudio se demuestra que aquellos alumnos que pasaban más "horas usando internet" tenían mayores puntuaciones en la variable de dificultades en salud mental. Dichos resultados son congruentes con el estudio realizado por Viñas (2009), donde se analizó el perfil psicológico de adolescentes españoles que 
pasaban muchas horas conectados a internet, los cuales obtuvieron altas puntuaciones en desesperanza y neuroticismo, pero bajas en extroversión y amabilidad. También se puede destacar el estudio de Agreda, Hinojo y Aznar (2016), llevado a cabo en la provincia de Granada (España), en el cual se mostró que la ansiedad o cambios en el estado de ánimo eran más frecuentes en aquellos adolescentes que utilizaban las tecnologías de una forma desmesurada. A nivel internacional, se encuentran otros estudios similares, como el de Rodríguez y Fernández (2014), en el que aquellos alumnos colombianos (11-15 años) que pasaban mucho tiempo usando redes sociales, mostraron mayores niveles de problemas externalizantes, tales como conducta agresiva, ruptura de reglas y problemas de atención. Resultados semejantes se observaron en otros trabajos internacionales donde se mostró cómo aquellos adolescentes que son adictos a internet o realizan un uso excesivo, son más propensos a tener comportamientos negativos (Ko, Yen, Liu, Huang, \& Yen, 2009) o tienen mayor probabilidad de consumir drogas, dificultades con el juego, peores resultados académicos y mayores dificultades psicosociales (Secades-Villa et al., 2014). En esta línea, estudios como el realizado por Cao, Sun, Wan, Hao y Tao (2011), pusieron de manifiesto que los menores que presentaban problemas de uso de internet, tenían más probabilidades de padecer síntomas psicosomáticos, entre los que se encuentran falta de energía física, disfunción fisiológica, inmunidad debilitada, síntomas emocionales, síntomas conductuales o problemas de adaptación social.

En relación con el análisis de la salud mental con las variables de tipo de centro, sexo, edad y curso académico, se encontró que no existen diferencias estadísticamente significativas excepto con edad. Por otra parte, la salud mental de los menores de 12 a 17 años no está asociada al "tipo de centro educativo" en el que estén matriculados. Este resultado puede deberse a que en los centros, ya sean públicos o concertados, se trabajan de la misma manera los temas relacionados con los problemas de dificultad en salud mental, como la hiperactividad, los problemas con los compañeros y de conducta, así como también aquellos vinculados a las emociones. También puede explicarse por el hecho de que en las ciudades pequeñas, como son las empleadas en nuestra investigación, no parece existir tanta diferencia entre los alumnos que asisten a centros públicos o privados. A pesar de ello, como se señaló anteriormente en la revisión teórica, Klanšček et al. (2014) encontraron en su estudio que el tipo de colegio ejerce una función significativa en la salud mental de los adolescentes. Es fundamental considerar que estos adolescentes se encontraban agrupados según el tipo de escuela secundaria existente en Eslovenia: escuelas industriales y artesanales, escuelas técnicas y relacionadas y, por último, otra más general, y no en función de que el centro fuera público o concertado. Por esta razón, se debe tener precaución a la hora de realizar comparaciones entre diferentes estudios, dependiendo de la clasificación de los tipos de centros establecida.

Es importante destacar, la ausencia de diferencias significativas entre la salud mental y la variable curso académico, frente a la existencia de ellas entre la salud mental y la variable edad. Una posible explicación se puede encontrar en la presencia de repetidores en los distintos cursos, quedando la muestra distribuida de forma más homogénea en los distintos cursos académicos que si se tiene en cuenta solo la edad. Así, en los resultados del presente estudio se encuentra que el grupo de alumnos de 12 años presentó puntuaciones significativamente menores en la variable dificultades en salud mental con respecto los demás grupos, excepto con los de 14 años. Estos hallazgos coinciden con lo revisado en la literatura científica, donde se encontró que el grupo de mayor edad, formado por los alumnos de 15 a 18 años, presentó mayores puntuaciones en el total de dificultades en comparación con el grupo de menor edad de 11 a 14 años (Arman et al., 2013). Datos similares se presentan en el estudio realizado por FajardoBullón, León-del-Barco, Felipe-Castaño, Polo del Río, \& Santos (2015), en el que señalan que, siguiendo a autores como Kessler et al. (2005), es bastante posible que se encuentren problemas 
Fernando Fajardo Bullón, Beatriz Burguillo Valverde, Benito León del Barco, et al.

de salud mental desde los 14 años en adelante, debido a que la mayor parte de trastornos mentales se detectan en torno a esa edad. Por el contrario, existen otros estudios como el de Van Roy et al. (2006), donde la puntuación total de dificultades en salud mental fue más alta en la adolescencia temprana de los niños (10 y 11 años), teniendo en cuenta que el rango de edad de la muestra fue de 10 a 19 años.

En cuanto a sexo y la edad, Ortuño-Sierra et al. (2014), encontraron en su estudio diferencias estadísticamente significativas en función del sexo y la edad, resultados coincidentes con los de la presente investigación respecto a la edad, pero divergentes en cuanto al sexo. Siguiendo a Fajardo-Bullón et al. (2012), las posibles diferencias halladas respecto al sexo en otros trabajos, puede justificarse por el estudio de patologías específicas en la investigación psicopatológica en salud mental, sin considerar una evaluación total. Sin embargo, este estudio no se centró solo en una patología específica o subescala, como pudieran ser los problemas de conducta o síntomas emocionales, sino que se hace una evaluación total a través de la puntuación de dificultades en salud mental. De aquí, se puede explicar el hecho de no encontrar diferencias significativas respecto a la variable sexo.

Como se mostró anteriormente, no logró alcanzar un consenso respecto a la edad, ya que existen diversos estudios que señalan un incremento de dificultades según va aumentando la edad, y otros que manifiestan lo opuesto. Por lo tanto, aunque todos coinciden en que la adolescencia cuenta con dificultades de salud mental, no se puede discriminar entre la adolescencia temprana o tardía (OrtuñoSierra et al., 2016). Tras analizar los resultados obtenidos, en los menores de 12-13 años se observa un incremento significativo de dificultades en salud mental, coincidiendo con la etapa de transición a la Educación Secundaria Obligatoria. Entre los 13 y los 17 años, igualmente se aprecia un crecimiento, pero se produce paulatinamente, excepto a los 14 años cuando no se observa ningún aumento. Existe una serie de factores relacionados con el tránsito de Educación Primaria a Secundaria: unos factores asociados a la propia adolescencia, en la que se dan determinados cambios físicos y psicológicos y otros vinculados al cambio de colegio, a tener que adaptarse a otro espacio nuevo para el adolescente (Cuberos, 2010). Sin embargo, a los 14 años, puede ocurrir un proceso de habituación al nuevo Centro, produciendo un descenso en sus dificultades de salud mental, pero aumentándose posteriormente, a su vez, por los factores psicosociales propios de la adolescencia.

\section{Limitaciones y futuras líneas de investigación}

Una de las limitaciones de este trabajo es el uso de una escala de salud mental que evalúa el estado del menor mediante autoinforme. Sería deseable contrastar esta información con versiones del SDQ-padres y SDQ-profesores que podrían evitar situaciones de deseabilidad social. De este modo, sería posible que se obtuvieran resultados y cifras todavía más llamativas y preocupantes. A su vez, sería interesante realizar futuras investigaciones que evalúen patologías con cuestionaros específicos para adolescentes, por ejemplo, de trastornos depresivos. Otras posibles reflexiones para futuros estudios serían las siguientes: se recomienda identificar al alumnado que no ha superado el curso académico y lo está repitiendo, como variable relevante en análisis de la edad y curso académico. A su vez, se recomiendan más trabajos que incorporen la variable centro público o privado. Por último, en futuras investigaciones habría que recoger información sobre el contenido de la actividad que desarrollan los menores durante las horas de uso de internet. Esa limitación también se ha producido en recientes estudios (El Asam et al., 2019) que indican la necesidad de trabajar, en futuras líneas de investigación, la importancia del contenido sobre lo que se consulta mientras se hace uso de internet. Por ejemplo, un aumento de horas de uso de internet puede deberse a periodos de mayor carga académica, que requieren cada vez más el uso de internet como herramienta de apoyo educativo, variable que debe ser tenida en cuenta para futuras investigaciones. 
En esta línea, surgen algunas preguntas que se deberían contemplar: ¿Qué contenidos son considerados adecuados y cuáles no? ¿Debemos valorar y categorizar la calidad de las horas dedicadas a internet? En este sentido, parece relevante el abordaje del uso de internet desde el área educativa, donde el docente tiene la responsabilidad de guiar a sus alumnos hacia un uso moderado y de calidad de las nuevas tecnologías (Upegui, 2017).

Por último, una vez conocida la relación entre el elevado número de horas de uso de internet y las dificultades que provoca en la salud mental (Ciarrochi et al., 2016), parece fundamental trabajar en programas de prevención, bien con familias o centros educativos, con la finalidad de disminuir el número elevado de horas que pasan los jóvenes conectados a internet y mejorar la salud mental. De este modo, se podría lograr un adecuado desarrollo personal y social de los adolescentes, desarrollando una buena salud en las futuras generaciones.

\section{Referencias}

Agreda, M., Hinojo, M., \& Aznar, I. (2016). Estudio evaluativo del impacto de las nuevas tecnologías en la juventud y adolescencia en la provincia de Granada, España. Revista Estudios Hemisféricos y Polares, 7(4), 61-77. Recuperado de http://www.revistaestudioshemisfericosy polares.cl/home/publicacion.php?id $=144$

Asociación para la Investigación de Medios de Comunicación. (2017). 19ํNavegantes en la red: encuesta AIMC a usuarios de internet 2016. Madrid: Autor.

Arman, S., Amel, A. K., \& Maracy, M. R. (2013). Comparison of parent adolescent scores on Strengths and Difficulties Questionnaire. Journal of Research in Medical Sciences, 18(6), 501-505. Recuperado de https://www.ncbi. nlm.nih.gov/pmc/articles/PMC3818621/

Cao, H., Sun, Y., Wan, Y., Hao, J., \& Tao, F. (2011). Problematic internet use in Chinese adolescents and its relation to psychosomatic symptoms and life satisfaction. BMC Public Health, 11 (1), 802. https://doi.org/10.1186/1471-2458-11-802

Ciarrochi, J., Parker, P., Sahdra, B., Marshall, S., Jackson, C., Gloster, A. T., \& Heaven, P. (2016). The development of compulsive internet use and mental health: A fouryear study of adolescence. Developmental Psychology, 52(2), 272-283. https://doi.org/ 10.1037/dev0000070

Cloquell, A. (2015). Usos sociales de internet entre los adolescentes españoles. Revista sobre la infancia y la adolescencia, 8, 1-14. ht tps://doi.org/10.4995/reinad.2015.3649

Cuberos, I. (2010). El tránsito: paso a paso desde Infantil hasta Secundaria. Revista Digital para Profesionales de la Enseñanza, 7, 1-7. Recuperado de https://www.feandalucia.cc oo.es/docuipdf.aspx? $\mathrm{d}=7046 \& s=$

Echeburúa, E. (2012). Factores de riesgo y factores de protección en la adicción a las nuevas tecnologías y redes sociales en jóvenes y adolescentes. Revista Española de Drogodependencias, 37(4), 435-447. Recuperado de https://dialnet.unirioja.es/se rvlet/articulo? codigo $=4113810$

El Asam, A., Samara, M., \& Terry, P. (2019). Problematic internet use and mental health among British children and adolescents. Addictive Behaviors, 90, 428-436. https://do i.org/10.1016/j.addbeh.2018.09.007

Fajardo-Bullón, F., Felipe-Castaño, E., Leóndel-Barco, B., Mendo Lázaro, S., Polo Del Río, M. I., \& Carroza, G. (2016). Harassment and mental health in Spanish children according to ENSE 2011. En J. L. Castejón Costa (Coord.), Psicología y educación: presente y futuro (pp. 1630-1636). Madrid: Asociación Científica de Psicología y Educación.

Fajardo-Bullón, F., León-del-Barco, B., FelipeCastaño, E., Polo del Río, M. I., \& Santos, E. (2015). Mental health in Spanish minors. Socioeducational variables. Salud Mental, 38(5), 329-335. https://doi.org/10. 17711/SM.0185-3325.2015.045

Fajardo-Bullón, F., León-del-Barco, B., FelipeCastaño, E., \& Santos, E. J. (2012). Mental 
Fernando Fajardo Bullón, Beatriz Burguillo Valverde, Benito León del Barco, et al.

health in the age group 4-15 years based on the results of the national survey of health 2006. Revista Española de Salud Pública, 86(4), 445-451. https://doi.org/10.4321/S1 $135-57272012000400011$

Fajardo-Bullón, F., Rasskin-Gutman, I., FelipeCastaño, E., Ribeiro dos Santos, E., \& León-del-Barco, B. (2017). Analysis of predictive factors on minors' mental health according to the Spanish National Health Survey. Brain Sciences, 7(10), 135. https://d oi.org/10.3390/brainsci7100135

Fenollar-Cortés, J., Calvo-Fernández, A., García-Sevilla, J., \& Cantó-Díez, T. J. (2016). La escala Strength and Difficulties Questionnaire (SDQ) como predictora del TDAH: comportamiento de las subescalas SDQ respecto a las dimensiones "Hiperactividad/ Impulsividad" e "Inatención" en una muestra clínica. Anales de Psicología, 32(2), 313-319. https://doi.org/10.6018/analesps. 32.2.203331

Fonseca-Pedrero, E., Paino, M., LemosGiráldez, S., \& Muñiz, J. (2011). Prevalencia de la sintomatología emocional y comportamental en adolescentes españoles a través del Strengths and Difficulties Questionnaire (SDQ). Revista de Psicopatología y Psicología Clínica, 16(1), 15-25. https://doi.org/10.5944/rppc.vol.16. num.1.2011.10348

Golpe, S., Isoma, M., Gómez., P., \& Rial, A. (2017). Uso problemático de internet y adolescentes: el deporte sí importa. RETOS. Nuevas Tendencias en Educación Física, Deporte y Recreación, 31, 52-57. Recuperado de https://dialnet.unirioja.es/se rvlet/articulo? codigo $=5841344$

Goodman, R. (1997). The Strengths and Difficulties Questionnaire: A research note. Journal of Child Psychology and Psychiatry, 38(5), 581-586. https://doi.org/1 0.1111/j.1469-7610.1997.tb01545.x

Işik, B., \& Ayaz Alkaya, S. (2017). Internet use and psychosocial health of school aged children. Psychiatry Research, 255,
204-208. https://doi.org/10.1016/j.psychres .2017 .05 .043

Kessler, R. C., Berglund, P., Demler, O., Jin, R., Merikangas, K. R., \& Walters, E. E. (2005). Lifetime prevalence and age-ofonset distributions of DSM-IV disorders in the National Comorbidity Survey Replication. Archives of General Psychiatry, 62 (6), 593-602. https://doi.org/10.1001/arc hpsyc.62.6.593

Klanšček, H. J., Žiberna, J., Korošec, A., Zurc, J., \& Albreht, T. (2014). Mental health inequalities in Slovenian 15-yearold adolescents explained by personal social position and family socioeconomic status. International Journal for Equity in Health, 13(26). https://doi.org/10.1186/1475-9276 $-13-26$

Ko, C. H., Yen, J. Y., Liu, S. C., Huang, C. F., \& Yen, C. F. (2009). The associations between aggressive behaviors and internet addiction and online activities in adolescents. Journal of Adolescent Health, 44(6), 598-605. https: //doi.org/10.1016/j.jadohealth.2008.11.011

Koskelainen, M., Sourander, A., \& Vauras, M. (2001). Self-reported strengths and difficulties in a community sample of Finnish adolescents. European Child $\mathbb{E}$ Adolescent Psychiatry, 10(3), 180-185. https ://doi.org/10.1007/s007870170024

León-del-Barco, B., Fajardo-Bullón, F., MendoLázaro, S., Rasskin-Gutman, I., \& IglesiasGallego, D.(2018). Impact of the familiar environment in 11-14-year-old minors' mental health. International Journal of Environmental Research and Public Health, 15(7),1314. https://doi.org/10.3390/ijerph1 5071314

Marciales, G. P., \& Cabra, F. (2011). Internet y pánico moral: revisión de la investigación sobre la interacción de niños y jóvenes con los nuevos medios. Universitas Psychologica, 10(3), 855-865. Recuperado de https://revistas.javeriana.edu.co/index.p hp/revPsycho/article/view/841

Martino, F. M. (2014). Las tecnologías de información y comunicación y el bienestar psicológico en la generación net. Hamut'ay, 
1(1), 39-51. https://doi.org/10.21503/hamu .v1i1.572

Mata, D., Suárez, A., Torres, C., Carro, A., \& Ortega, E. (2009). Uso del Cuestionario de Capacidades y Dificultades (SDQ) como instrumento de cribado de trastornos psiquiátricos en la consulta de pediatría de Atención Primaria. Boletín de Pediatría, 49(209), 259-262. Recuperado de https://www.sccalp.org/documents/0000 /1468/BolPediatr2009 49 259-262.pdf

Montero, I., \& León, O. G. (2007). A guide for naming research studies in Psychology. International Journal of Clinical and Health Psychology, 7(3), 847-862. Recuperado de h ttp://www.aepc.es/ijchp/GNEIP07_es.pdf

Muris, P., Meesters, C., \& Van den Berg, F. (2003). The strengths and difficulties questionnaire (SDQ): Further evidence for its reliability and validity in a community sample of Dutch children and adolescents. European Child 8 Adolescent Psychiatry, 12(1), 1-8. https://doi.org/10.1007/s00787003-0298-2

Ortuño-Sierra, J., Chocarro, E., FonsecaPedrero, E., Sastre i Riba, S., \& Muñiz, J. (2015). The assessment of emotional and behavioural problems: Internal structure of The Strengths and Difficulties Questionnaire. International Journal of Clinical and Health Psychology, 15(3), 265-273. https://doi.org/10.1016/j.ij chp.2015.05.005

Ortuño-Sierra, J., Fonseca-Pedrero, E., Inchausti, F., \& Sastre i Riba, S. (2016). Evaluación de dificultades emocionales y comportamentales en población infantojuvenil: el Cuestionario de Capacidades y Dificultades (SDQ). Papeles del Psicólogo, 37(1), 14-26. Recuperado de http://www.p apelesdelpsicologo.es/pdf/2658.pdf

Ortuño-Sierra, J., Fonseca-Pedrero, E., Paino, M., \& Aritio-Solana, R. (2014). Prevalencia de síntomas emocionales y comportamentales en adolescentes españoles. Revista de Psiquiatría y Salud Mental, 7(3), 121-130. https://doi.org/10.1 016/j.rpsm.2013.12.003
Rodríguez, A. P., \& Fernández, A. (2014). Relación entre el tiempo de uso de las redes sociales en internet y la salud mental en adolescentes colombianos. Acta Colombiana de Psicología, 17(1), 131-140. h ttps://doi.org/10.14718/ACP.2014.17.1.13

Rodríguez, P. J., \& Pérez, E. (2012). Utilización de cuestionarios/ test psicométricos en Pediatría de Atención Primaria. Pediatría Integral, 16(10). 810.e1-810.e7. Recuperado de https://www.pediatriaintegral.es/numer os-anteriores/publicacion-2012-12/utilizaci on-de-cuestionariostest-psicometricos-enpediatria-de-atencion-primaria/

Rothenberger, A., Becker, A., Erhart, M., Wille, N., Ravens-Sieberer, U., \& Bella Study Group. (2008). Psychometric properties of the parent strengths and difficulties questionnaire in the general population of German children and adolescents: Results of the BELLA study. European Child $\mathcal{E}$ Adolescent Psychiatry, 17(1), 99-105. https:/ /doi.org/10.1007/s00787-008-1011-2

Secades-Villa, R., Calafat, A., FernándezHermida, J. R., Duch, M., Montse, J., Skärstrand, E., ... Talic, S. (2014). Duration of internet use and adverse psychosocial effects among European adolescents. Adicciones, 26(3), 247-253. Recuperado de http://www.irefrea.eu/uploads/PDF/Sec ades_etal-2014_Internet_PsychologicalEff ects.pdf

Upegui, M. A. (2017). El consumo tecnológico de los jóvenes. Un desafío para los docentes. Colección Académica de Ciencias Sociales, 4(1), 51-60. Recuperado de https://revistas.upb.edu.co/index.php/ci enciassociales/article/view/7162/6539

Van Roy, B., Grøholt, B., Heyerdahl, S., \& Clench-Aas, J. (2006). Self-reported strengths and difficulties in a large Norwegian population 10-19 years: Age and gender specific results of the extended SDQ-Questionnaire. European Child 8 Adolescent Psychiatry, 15(4), 189-198. https ://doi.org/10.1007/s00787-005-0521-4 
Fernando Fajardo Bullón, Beatriz Burguillo Valverde, Benito León del Barco, et al.

Viñas, F. (2009). Uso autoinformado de internet en adolescentes: perfil psicológico de un uso elevado de red. International Journal of Psychology and Psychological Therapy, 9(1), 109-122. Recuperado de http://www.infoco p.es/view_article.asp?id $=2296$

Yao, S., Zhang, C., Zhu, X., Jing, X., McWhinnie, C. M., \& Abela, J. R. (2009). Measuring adolescent psychopathology: Psychometric properties of the self-report strengths and difficulties questionnaire in a sample of Chinese adolescents. Journal of Adolescent Health, 45(1), 55-62. https://doi.org/10.101 6/j.jadohealth.2008.11.006

Zubeidat, I., Fernández-Parra, A., Ortega, J., Vallejo, M. Á., \& Sierra, J. C. (2009). Características psicosociales y psicopatológicas en una muestra de adolescentes españoles a partir del Youth Self-Report/11-18. Anales de Psicología, 25(1), 60-69. Recuperado de https://psycne t.apa.org/record/2009-08527-007

\section{Notas}

* Artículo de investigación. 